\title{
Acknowledgment of JAISE reviewers in 2019
}

Over the past eleven years of its life, our Journal has been supported by a large number of colleagues who contributed with their time and expertise to assess the quality of the submissions to JAISE and helped decide which papers qualify to be published. These reviewers are an important part of the JAISE community and we would like to explicitly thank all of them for their valuable contributions.

The effort of reviewers often remains unnoticed in the community served by a journal, especially in a blind review system. Since six years ago, we have been acknowledging the participation of our reviewers in the making of JAISE.

As a second step towards making our gratitude explicit and highlighting the importance of the contributions made by our reviewers, we have also implemented the practice of selecting two reviewers each year who have consistently provided detailed and quality reviews and inviting them to serve as part of the Editorial Board of JAISE.

The list of reviewers in 2019 includes: ${ }^{1}$

Soheila Abrishami, Hina Afreen, Aziz Ahmad, Muhammad Alam, Sadi Alawadi, M. Aleem, Hande Alemdar, Murad Ali, Saad Ali, Alaa Aljanaby, Shayma Alkobaisi, Juan Antonio Álvarez-García, Inmaculada Ayala, Arshia Azam Azam, Thierry badard, Costin Badica, Serkan Balli, Sadiq Bugati, Davide Carneiro, Yoong Choon Chang, Wei Chen, Stefano Chessa, Gabriele Civitarese, Pau Climent-Perez, Nuno Costa, Roberto da Silva, Andreea Danielescu, Aldo Franco Dragoni, Carlos Duarte, Marco Eichelberg, Rose Faghih, João Filipe Ferreira, Francesco Furfari, Jose Gines Gimenez Manuel, Michele Girolami, Carles Gomez, alberto gotta, Bjoern Gottfried, Gregor Grasselli, David Griol, André Hirakawa, R. Hussain, M. Imran, Alexandros Iosifidis, Waqar Jaffry, Ozgur Kafali, Omprakash Kaiwartya, Rafaqut Kazmi, Rafaqut Kazmi, Rafaqut Kazmi, Shehroz Khan, Taimoor Khan, Zaheer Khan, Shehroz Khan, Alexander Kocian, Tine Kolenik, Leon Lei, Barbara Lep- orini, Jie Li, Zhenglong Li, Ray Z.X. Li, Yaqing Liu, Xi Long, António Lopes, Jin Lu, Davoli Luca, K.S. Lui, José Machado, Felipe Masculo, Georgios Meditskos, Antonios Michalas, Popescu Mihail, Peter Mikulecky, Alberto Monge Roffarello, Hector Moretón, Bushra Mughal, Nazrin Muhammad, Georgios Mylonas, Tatsuo Nakajima, Anand Nayyar, Michel Oey, Paulo Oliveira, Filippo Palumbo, Parvaneh Parvin, IonelBujorel Pavaloiu, Adam Pease, Thinagaran Perumal, Francesco Piccialli, Bastien Poggi, Andrea Prati, Davy Preuveneers, Francesca Pulina, Mario Quinde, Shabana Ramzan, Alicia Rebollar, George Roussos, Dario Salvi, Rubén San-Segundo, Muhammad Sarfraz, Bareera Sarwar, Hina Sattar, Volkan Senyurek, Ishwar Sethi, Syed Attique Shah, Yiru Shen, Daniele Sora, Gulrez T., Warner ten Kate, Qinglin Tian, Alessandro Tognetti, Joaquin Torres-Sospedra, Chrisa Tsinaraki, Akgül Özgür Umut, Paulo Urbano, Maria Valera Espina, Egon van den Broek, Tim Van Hamme, Andrea Vitaletti, Jan Vox, Kevin Wang, Jose Wielki, Zhenyi Xu, Poonam Yadav, Salisu Yahaya, Sule Yildirim, Tongda Zhang, Junpei Zhong, Zhenxing Zhou, Jernej Zupancic.

\footnotetext{
${ }^{1}$ Names appear as they have been entered in the system.
} 